\title{
Erratum to: The Differential Contributions of Conceptual Representation Format and Language Structure to Levels of Semantic Abstraction Capacity
}

\author{
Guido Gainotti ${ }^{1,2}$ (D)
}

Published online: 9 June 2017

(C) Springer Science+Business Media New York 2017

Erratum to: Neuropsychol Rev

DOI 10.1007/s11065-016-9339-8

The original version of this article contained mistake in the author group. The author's given and family names were interchanged. The correct name is shown above. The original article has been corrected.

The online version of the original article can be found at http://dx.doi. org/10.1007/s11065-016-9339-8

Guido Gainotti

gainotti@rm.unicatt.it

1 Department of Neurosciences and Center for Neuropsychological Research, Institute of Neurology, Policlinico Gemelli, Catholic University of Rome, Largo A. Gemelli, 8, 00168 Rome, Italy

2 Department of Clinical and Behavioral Neurology, IRCCS, Fondazione Santa Lucia, Rome, Italy 